\title{
Temporal cavity solitons and frequency combs via quantum interference
}

\author{
Gian-Luca Oppo $\odot,{ }^{1, *}$ David Grant, ${ }^{1}$ and Mansour Eslami $\odot^{2}$ \\ ${ }^{1}$ SUPA and Department of Physics, University of Strathclyde, Glasgow G4 ONG, Scotland, United Kingdom \\ ${ }^{2}$ Department of Physics, University of Guilan, P.O. Box 41335-1914, Rasht, Iran
}

(Received 17 April 2021; accepted 3 January 2022; published 20 January 2022)

\begin{abstract}
Temporal cavity solitons in ring microresonators provide broad and controllable generation of frequency combs with applications in frequency standards and precise atomic clocks. Three-level media in the $\Lambda$ configuration inside microresonators displaying electromagnetically induced transparency can be used for the generation of temporal cavity solitons and frequency combs in the presence of anomalous dispersion and two external driving fields close to resonance. Here domain walls separating regions of two dark states due to quantum interference correspond to realizations of stimulated Raman adiabatic passage without input pulses. With no need of modulational instabilities, bright temporal cavity solitons and frequency combs are formed when these domain walls lock with each other. Wide stability ranges, close to resonance operation, and the optimal shape of the cavity solitons due to three-level quantum interference can make them preferable to those in two-level media.
\end{abstract}

DOI: 10.1103/PhysRevA.105.L011501

Frequency combs for precision optical synthesizing have come a long way in the past two decades from mode-locked lasers with cavity lengths between $30 \mathrm{~cm}$ and $3 \mathrm{~m}$ to chip-scale sources based on microresonators [1,2]. When operating at low loss, microresonators provide long interaction times and can excite large nonlinearities. Considerable attention was paid to the theoretical and experimental development of frequency combs in high- $Q$ optical resonators operating away from material resonances via Kerr nonlinearities. Temporal cavity solitons (TCSs) [3], the analog of transverse (diffraction) cavity solitons $[4,5]$ in the longitudinal (dispersion) domain, are key nonlinear solutions for the generation of broad frequency combs in passive [6,7] and active [8] media. These devices benefit from a variety of technological merits including low-energy requirements and robust structures that can be integrated on a chip [1,9]. Here the longitudinal coordinate that spans the resonator length is replaced by a fast-time variable when considering the group-velocity dispersion of the propagating light.

At the same time, substantial progress has been made in the realm of quantum interference phenomena for the manipulation of the optical response of a medium close to material resonances. Electromagnetically induced transparency (EIT) is one such phenomenon where the optical response of the material can be controlled by external electromagnetic fields to forbid, for example, absorption or to enhance the refractive index [10]. In this transparency window, dispersion properties are also strongly modified, motivating many applications such as slow light and stored light [11]. The underlying mechanism is the quantum interference effect that destructively couples the transition amplitudes of different excitation pathways [10]. Since then, mircoresonators have become an interesting platform for the realization of EIT leading to solid-state-based

\footnotetext{
*g.1.oppo@strath.ac.uk
}

technologies and new applications in sensing and field enhancement [12,13]. Recently, EIT resonance line shapes have been found in a variety of optical cavities [14] including microring resonators in silicon-on-insulator chips with air holes [15]. On the theoretical side, optical cavities with three-level media displaying EIT have also been studied for the onset and stability of various transverse structures, from patterns to diffractive cavity solitons [16-18].

Yet to be studied are the fast-time EIT features for microresonators in the presence of longitudinal dispersion. It is the aim of this Letter to show that quantum interference coupled with anomalous dispersion in microresonators displaying EIT can lead to bistability of dark states, stable domain walls (DWs) between these states, trapped stimulated Raman adiabatic passage (STIRAP) [19] schemes with no input pulses, novel TCSs, and frequency combs induced by quantum interference and EIT.

We consider a ring microresonator as displayed in Fig. 1(a) filled with a three-level medium in the $\Lambda$ configuration [Fig. 1(b)] with the transition between the two lower levels $|1\rangle$ and $|2\rangle$ not dipole allowed. There are two optical beams interacting with the medium, the resonated field $E$ circulating in the ring cavity under the external $\mathrm{cw}$ driving $P$ and the nonresonated field $E_{2}$ of constant intensity and at resonance with the transition between levels $|2\rangle$ and $|3\rangle$. The field $E$ is considered here to be detuned by $\Delta$ from the resonance of levels $|1\rangle$ and $|3\rangle$. Note that the results presented below extend to wide ranges of $\Delta$, to fields $E_{2}$ away from resonance, and slow relaxations of the level $|2\rangle$ to level $|1\rangle$. In the case of a fast response medium, the dynamics of the field $E$ in the ring resonator is described by [20]

$$
\partial_{t} E=P-(1+i \theta) E+i(2 C) R_{13}+i \partial_{\tau^{2}} E,
$$

where $t$ is the slow time over several round-trips in the cavity, $P$ is the amplitude of the input pump, $\theta$ is the cavity detuning from the input frequency, and $2 C$ is the cooperativity 


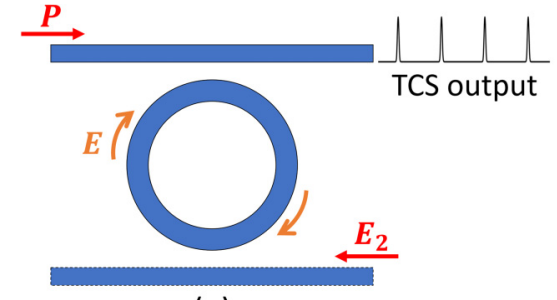

(a)

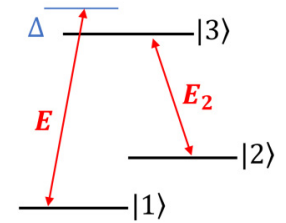

(b)

FIG. 1. (a) Schematic representation of the dispersive ringresonator device described by Eq. (1). (b) A $\Lambda$-type three-energylevel configuration. Symbols are described in the text.

parameter of the light-matter coupling that is proportional to the dipole moment of the transition between levels $|1\rangle$ and $|3\rangle$. In [16-18] we focused on the transverse diffractive case, while here we investigate the anomalous group-velocity-dispersion case with a longitudinal variable $\tau$, the fast time, defined in a reference frame moving at the group velocity of the light at the driving wavelength. Here $R_{13}$ is the density matrix element in the Lindblad master equation given by $[16,21]$

$$
\begin{aligned}
R_{13}= & \chi\left(|E|^{2}\right) E=-\frac{\Delta\left|E_{2}\right|^{2}\left(\left|E_{2}\right|^{2}+|E|^{2}-\Delta^{2}-i \Delta\right)}{D} E, \\
D= & \left(\left|E_{2}\right|^{2}+|E|^{2}\right)^{3} \\
& +\Delta^{2}\left(\left|E_{2}\right|^{2}+|E|^{2}+4\left|E_{2}\right|^{2}|E|^{2}+\Delta^{2}\left|E_{2}\right|^{2}-2\left|E_{2}\right|^{4}\right) .
\end{aligned}
$$

The detuning $\Delta$ can be scanned by changing the frequency of the input laser. This results in changes of the cavity detuning $\theta$ as well. It is possible, however, to scan the cavity detuning $\theta$ without affecting $\Delta$ by operating on cavity features such as its length. This is different from the two-level medium case where the medium response is basically unaffected by the changes in the frequency of the input beam because one is operating far from the medium resonance. Equation (2) is derived from [21] in [22] for a three-level medium in the $\Lambda$ configuration together with the Lugiato-Lefever equation for a two-level medium in the Kerr approximation [23] for a comparison. In the following we discuss cavity frequency scans where the input frequency and consequently $\Delta$ are kept fixed.

The intensities $\left|E_{s}\right|^{2}$ of the homogeneous stationary solutions (HSSs) are obtained from the equation

$$
|P|^{2}=\left\{[1-2 C \operatorname{Im}(\chi)]^{2}+[\theta+2 C \operatorname{Re}(\chi)]^{2}\right\}\left|E_{s}\right|^{2},
$$

where $\operatorname{Re}(\chi)$ and $\operatorname{Im}(\chi)$ are the real and imaginary parts of the complex susceptibility of Eq. (2). For small values of the intensity of the second field $E_{2}$, one observes a typical bistability close to cavity resonance which is however strongly enhanced by the quantum interference and splits into a closed bubble and a low-intensity branch as shown in Fig. 2(a). It is possible to obtain the stability of the HSS through standard linearization techniques. In Fig. 2(a), for $\left|E_{2}\right|^{2}=0.1$, $P=2.5,2 C=35$, and $\Delta=0.66$ we see that the low-intensity HSS branch is always stable, the lower part of the bubble is always unstable to homogeneous perturbations (red dashed curve), and the high-intensity HSS branch is stable for $\theta>$ -1.6 and unstable to modulated perturbations (patterns) for $-2.6<\theta<-1.6$. The HSS expressions of the probability of
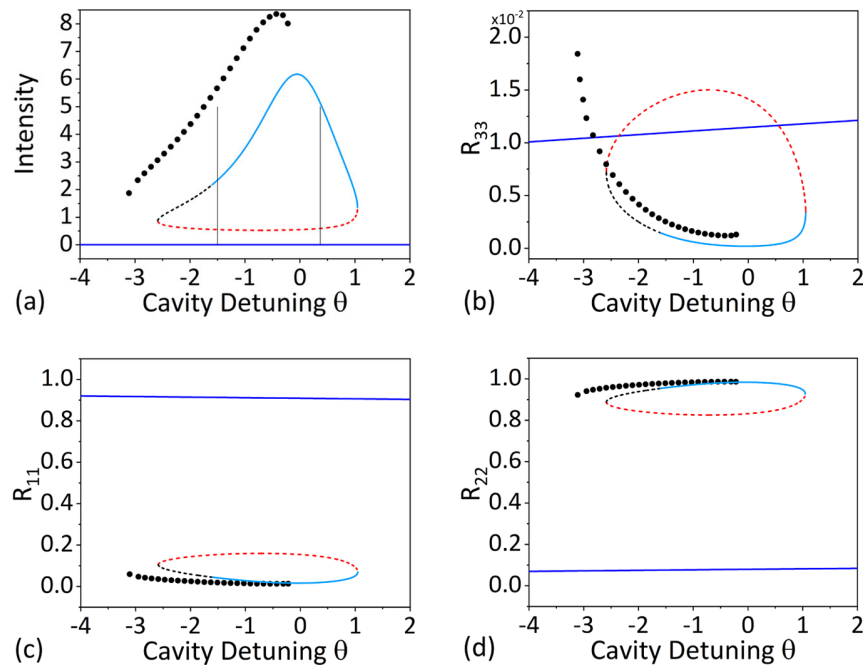

FIG. 2. (a) Intensity, (b) $R_{33}$, (c) $R_{11}$, and (d) $R_{22}$ for the HSSs of Eq. (1) for $\left|E_{2}\right|^{2}=0.1, P=2.5,2 C=35$, and $\Delta=0.66$ when changing the cavity detuning $\theta$. Light and dark blue solid lines are for stable HSSs, red and black dashed lines are for unstable HSSs, and black dots are for TCS peaks. The vertical lines in (a) identify the range where moving or stationary DWs connecting two stable HSSs are found.

occupancy of each of the three levels are given by

$$
\begin{aligned}
& R_{11}=1-R_{22}-R_{33}, \\
& R_{22}=\frac{\left|E_{s}\right|^{2}\left[\left(\left|E_{s}\right|^{2}+\left|E_{2}\right|^{2}\right)^{2}+\Delta^{2}\left(1+\left|E_{2}\right|^{2}\right)\right]}{D}, \\
& R_{33}=\frac{2 \Delta^{2}\left|E_{s}\right|^{2}\left|E_{2}\right|^{2}}{D},
\end{aligned}
$$

where $D$ is the denominator appearing in Eq. (2) with the HSS intensities replacing $|E|^{2}$ [plotted in Figs. 2(b)-2(d)]. The first key observation is that for a generic medium and cavity configurations close to medium resonance, coherent population trapping (CPT) and extremely low values of the probability of occupancy of the excited state $|3\rangle$ are observed [see values of $R_{33}$ below $2 \%$ in Fig. 2(b)]. From Figs. 2(c) and 2(d) we see that the stable low- (high-)intensity HSS corresponds to maintaining more than $90 \%$ of the population in level $|1\rangle$ (level $|2\rangle$ ). This is a remarkable resonant coupling between the medium properties and the cavity confinement induced by the quantum interference in the three-level medium leading to the almost exclusive population of dark states $\mathcal{D}=$ $\cos (\beta)|1\rangle-\sin (\beta)|2\rangle$, where $\beta$ is the mixing angle with values close to either zero or $\pi / 2$.

To understand the mechanisms behind these effects, we have plotted in Fig. 3 the chromatic dispersion and absorption properties of the $|1\rangle-|3\rangle$ transition of the $\Lambda$ system in the two bistable HSSs at cavity resonance when changing the detuning $\Delta$. In the low-intensity branch, chromatic dispersion shifts the input frequency and removes the effect of the cavity resonance. The absorption has a typical EIT shape and is ineffective over a broad band of detunings $\Delta$, thus explaining why $90 \%$ of the population remains confined to level $|1\rangle$. The high-intensity branch has almost zero dispersion, is strongly affected by the cavity resonance [note the peak in Fig. 2(a) 


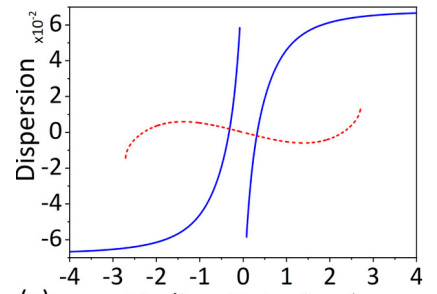

(a)
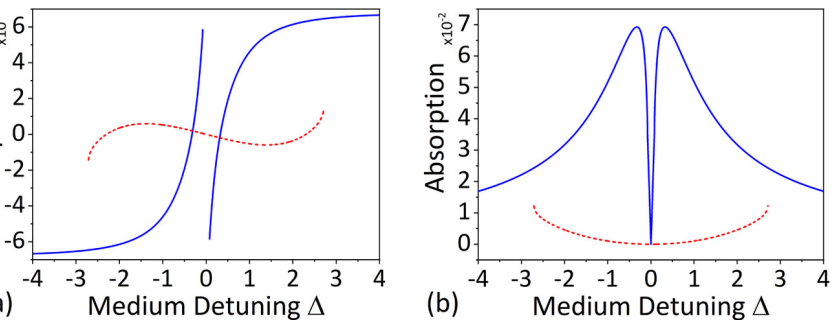

FIG. 3. (a) Chromatic dispersion $\operatorname{Re}\left(R_{13}\right)$ and (b) absorption $\operatorname{Im}\left(R_{13}\right)$ versus the material detuning $\Delta$ for the $|1\rangle-|3\rangle$ transition for $\theta=0,\left|E_{2}\right|^{2}=0.1$, and $P=2.5$. Blue solid (red dashed) lines correspond to the low- (high-)intensity branch of the bistable HSSs.

close to $\theta=0$ ], and displays a broadband EIT due to quantum interference.

On both sides of cavity resonance, we observe bistability of the low- and high-intensity branches of the HSSs. We then investigate the existence and stability of heteroclinic solutions that move from the low-intensity branch to the highintensity branch, and vice versa, during a round-trip of the cavity. Steep kinks from one branch to the other are known as domain walls (or switching waves [24] in the absence of an exchange symmetry) in analogy with magnetic systems [25]. For example, in magnetic nanomaterials [26], flexible metamaterials [27], and nematic liquid crystals [28], DWs have a typical hyperbolic tangent shape, annihilate each other when the kink and antikink collide, and require defects for their pinning. In nonlinear optics, DWs have been described in optical parametric oscillators [29-31] and in Kerr resonators with two orthogonal polarizations [32,33], while switching waves have been investigated in Kerr resonators in [34-37]. By tuning $\theta$ inside the range of $-1.5<\theta<0.37$ where DWs connecting two stable HSS are found [Fig. 2(a)], it is possible to find Maxwell points, labeled $\theta_{M}$, where upward (kink) and downward (antikink) DWs exist and are stationary at many separation distances as shown, for example, in Fig. 4, where we consider microresonators with a free spectral range of around $140 \mathrm{GHz}$, input powers of a few milliwatts, and realistic detunings in agreement with realizations of
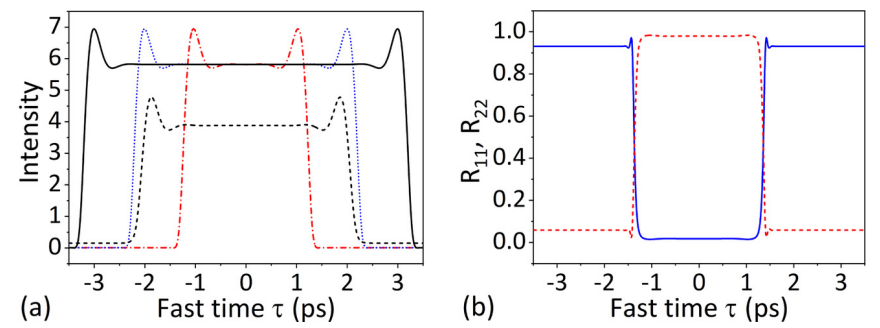

FIG. 4. Asymptotic states from numerical integration of Eq. (1). (a) Intensity profiles of two DWs at stable distances for $\theta_{M}=$ $-0.305,\left|E_{2}\right|^{2}=0.1, P=2.5,2 C=35$, and $\Delta=0.66$ (black solid, blue dotted, and red dash-dotted lines correspond to different initial separations of the kink and antikink DWs) and for $\theta_{M}=-0.37$, $\left|E_{2}\right|^{2}=1.0, P=2.0,2 C=35$, and $\Delta=0.2$ (black dashed line). (b) Fast-time distributions of occupancy probabilities of level $|1\rangle$, $R_{11}$ (blue solid line), and level $|2\rangle, R_{22}$ (red dashed line), for the case of the red dash-dotted line of (a).
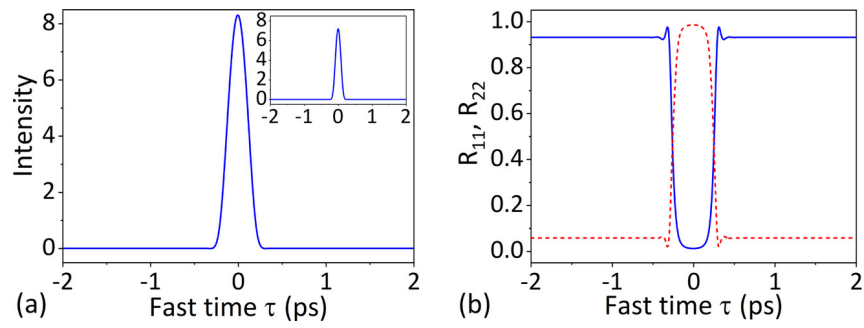

FIG. 5. Asymptotic states from numerical integration of Eq. (1). (a) Intensity profile of a quantum interference TCS for $\theta=-0.5$ ( $\theta=-1.0$ in the inset), $\left|E_{2}\right|^{2}=0.1, P=2.5,2 C=35$, and $\Delta=$ 0.66 . According to the chosen free spectral range of around $140 \mathrm{GHz}$, the FWHM size of the TCS is about 0.27 ps (0.18 ps in the inset). (b) Fast-time distributions of occupancy probabilities of level $|1\rangle$, $R_{11}$ (blue solid line), and level $|2\rangle, R_{22}$ (red dashed line).

silicon-on-insulators devices [38]. Stationary and moving DW solutions coexist with stable HSSs inside the two vertical lines of Fig. 2(a). When moving across a DW inside the resonator, the probability of occupancy goes from being in state $|1\rangle$ (low output intensity) to state $|2\rangle$ (high output intensity) as displayed in Fig. 4(b), while the population of level $|3\rangle$ remains well below $1 \%$ due to CPT and EIT. More than $90 \%$ of the population of level $|1\rangle$ can be transferred to level $|2\rangle$ when moving from one side of the DW to the other. This is nothing other than a cavity-enhanced STIRAP process, the powerful method for efficient and selective transfer of population between two quantum states [19]. Stimulated Raman adiabatic passage has an enormous number of applications [39] and is generally based on the sequence of two gated pulses of laser light with appropriate Rabi frequency (i.e., intensity) profiles. The DW-based STIRAP presented here requires no pulsed light at the input. Once the DWs are formed by an initial perturbation, they keep circulating in the resonator with one part of the resonator residing in one of the two (dark) quantum states, leaving the other part in the second of the two (dark) quantum states. It is interesting to note that stable DWs correspond to a continuous-wave realization of STIRAP since the input pumps $P$ and $E_{2}$ are not pulsed as it is standard in STIRAP configurations [19,39].

Domain walls are not the only localized structures due to quantum interference in this system. Away from the values of the cavity detuning $\theta_{M}$ where the DWs maintain a fixed distance, the DWs move away from (towards) each other for $\theta>\theta_{M}\left(\theta<\theta_{M}\right)$ when $\Delta=0.66$ and $\left|E_{2}\right|^{2}=0.1$. When compared to DWs in solid-state physics of hyperbolic tangent shape [26-28], our optical DWs display large intensity peaks when approaching the upper HSS because of group-velocity dispersion instead of diffusion. These local peaks are essential for stable TCS formed by locked DWs without the requirement of pinning defects. If the cavity detuning $\theta$ is smaller than $\theta_{M}$, two DWs move towards each other and, in view of the large oscillation close to the top of the DWs, they lock and form a TCS without requiring modulational instabilities in a way similar to what was described in $[30,31]$ for parametric oscillators. For $\theta=-0.5$, for example, the low-intensity HSS expands in the high-intensity HSS until the DWs lock and form a quantum interference TCS [see Fig. 5(a)] with an underlying STIRAP process of more than $97 \%$ since the prob- 

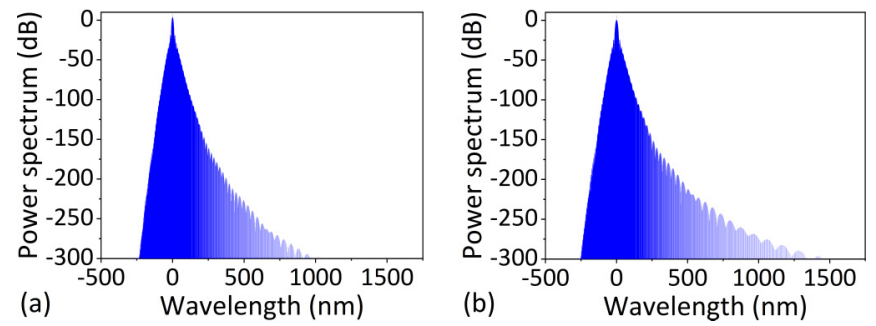

FIG. 6. Spectrum of the locked DW TCS of Fig. 5 for (a) $\theta=$ -0.5 and (b) $\theta=-1.0$ after subtraction of the carrier wavelength of the input light.

ability of occupancy of state $|1\rangle$ is very high in the TCS tails and the probability of occupancy of state $|2\rangle$ is very high at the TCS peak [see Fig. 5(b)]. The TCS peak intensities and occupancy probabilities over the interval of TCS existence versus the cavity detuning parameter are reported as black circles in Fig. 2. Typical FWHM sizes of TCSs due to quantum interference are expected to be in the subpicosecond regime. Domain walls and TCSs formed by locked DWs are localized quantum interference structures robust to noise perturbations of more than $40 \%$ of their amplitudes, do not require modulational instabilities, and exist in wide ranges of the parameter space of the model equations, making them likely to be implemented experimentally. It is important to note that stable and moving DWs have been observed experimentally for two-level media with normal dispersion $[32,33]$ in regimes not affected by the presence of pattern solutions. The quantum interference DWs and TCSs presented here operate instead in the anomalous dispersion regime.

Quantum interference TCSs formed by locked DWs have a very high contrast (visibility) since their tails are anchored on the low-intensity branch. When compared with their counterpart in Kerr two-level media [22], they require four times lower input powers (order of a few milliwatts) and present almost no local modulations at the bottom of the peaks, mak- ing them excellent candidates for the generation of frequency combs. Figure 6 shows two examples of frequency combs for the values of $\theta=-0.5$ and $\theta=-1.0$. In the first case we have a higher peak intensity TCS with higher definition but narrower spectrum, while in the second case we have a narrower TCS resulting in a broader spectrum. These are excellent realizations of frequency combs made of quantum interference dark states and cw STIRAP.

Quantum interference between separate two-level transitions leads to CPT, EIT, and the population of dark states in three-level $\Lambda$ configurations. Here we have shown that quantum interference can also be responsible for stable DWs and TCSs due to the locking of DWs in microresonators driven by two external fields close to the medium resonances and in the presence of anomalous group-velocity dispersion. The DWs separate two dark states inside the optical cavity where the medium is almost exclusively in one or the other of the two ground-state energy levels. This phenomenon corresponds to the realization of cavity STIRAP with no input pulses.

In contrast to two-level media, TCSs via quantum interference and anomalous dispersion can occur without modulational instabilities and close to medium and cavity resonances due to EIT, very low absorption, and enhanced nonlinear features. Close to resonance TCSs have optimal shapes for nonlinear absorbers owing to their high peak intensities, very low backgrounds, and almost no oscillation in the tails. For these reasons, TCSs due to quantum interference can be optimal candidates for the generation of novel microresonator frequency combs with applications in frequency standards, optical communications, and high-resolution spectroscopy. Our DWs and TCSs are robust features and are expected for wide ranges of input powers, pulsed regimes, medium responses, and detunings. Dynamical TCSs, broadening of frequency combs, generalizations to normal dispersion, and detuned fields $E_{2}$ of large Rabi frequencies leading to Fano-like resonances as well as quantum interference in $V$ and ladder three-level media in microresonators are left for future investigation.
[1] A. Pasquazi, M. Peccianti, L. Razzari, D. J. Moss, S. Coen, M. Erkintalo, Y. K. Chembo, T. Hansson, S. Wabnitz, P. Del'Haye, X. Xue, A. M. Weiner, and R. Morandotti, Micro-combs: A novel generation of optical sources, Phys. Rep. 729, 1 (2018).

[2] T. Fortier and E. Baumann, 20 years of developments in optical frequency comb technology and applications, Commun. Phys. 2, 153 (2019).

[3] F. Leo, S. Coen, P. Kockaert, S.-P. Gorza, P. Emplit, and M. Haelterman, Temporal cavity solitons in one-dimensional Kerr media as bits in an all-optical buffer, Nat. Photon. 4, 471 (2010).

[4] A. J. Scroggie, W. J. Firth, G. S. McDonald, M. Tlidi, R. Lefever, and L. A. Lugiato, Pattern formation in a passive Kerr cavity, Chaos Soliton. Fractal. 4, 1323 (1994).

[5] T. Ackemann, W. Firth, and G.-L. Oppo, Fundamentals and applications of spatial dissipative solitons in photonic devices, Adv. At. Mol. Opt. Phys. 57, 323 (2009).
[6] T. Herr, V. Brasch, J. D. Jost, C. Y. Wang, N. M. Kondratiev, M. L. Gorodetsky, and T. J. Kippenberg, Temporal solitons in optical microresonators, Nat. Photon. 8, 145 (2014).

[7] T. J. Kippenberg, A. L. Gaeta, M. Lipson, and M. L. Gorodetsky, Dissipative Kerr solitons in optical microresonators, Science 361, 567 (2018).

[8] H. Bao, A. Cooper, M. Rowley, L. Di Lauro, J. S. Totero Gongora, S. T. Chu, B. E. Little, G.-L. Oppo, R. Morandotti, D. J. Moss, B. Wetzel, M. Peccianti, and A. Pasquazi, Laser cavity-soliton microcombs, Nat. Photon. 13, 384 (2019).

[9] Y. K. Chembo, Kerr optical frequency combs: Theory, applications and perspectives, Nanophotonics 5, 214 (2016).

[10] M. Fleischhauer, A. Imamoglu, and J. P. Marangos, Electromagnetically induced transparency: Optics in coherent media, Rev. Mod. Phys. 77, 633 (2005). 
[11] I. Novikova, R. L. Walsworth, and Y. Xiao, Electromagnetically induced transparency-based slow and stored light in warm atoms, Laser Photon. Rev. 6, 333 (2012).

[12] Y.-F. Xiao, L. He, J. Zhu, and L. Yang, Electromagnetically induced transparency-like effect in a single polydimethylsiloxane-coated silica microtoroid, Appl. Phys. Lett. 94, 231115 (2009).

[13] Y.-C. Liu, B.-B. Li, and Y.-F. Xiao, Electromagnetically induced transparency in optical microcavities, Nanophotonics $\mathbf{6}$, 789 (2017).

[14] H. Qin, M. Ding, and Y. Yin, Induced transparency with optical cavities, Adv. Photon. Res. 1, 2000009 (2020).

[15] L. Gu, H. Fang, J. Li, L. Fang, S. J. Chua, J. Zhao, and X. Gan, A compact structure for realizing Lorentzian, Fano, and electromagnetically induced transparency resonance lineshapes in a microring resonator, Nanophotonics 8, 841 (2018).

[16] G.-L. Oppo, Complex spatial structures due to atomic coherence, J. Mod. Opt. 57, 1408 (2010).

[17] M. Eslami, R. Kheradmand, D. McArthur, and G.-L. Oppo, Complex structures in media displaying electromagnetically induced transparency: Pattern multistability and competition, Phys. Rev. A 90, 023840 (2014).

[18] M. Eslami, R. Kheradmand, and G.-L. Oppo, Complex structures in cavities with media displaying EIT: Coexistence, defects and selection mechanism, J. Phys. B 53, 075402 (2020).

[19] N. V. Vitanov, A. A. Rangelov, B. W. Shore, and K. Bergmann, Stimulated Raman adiabatic passage in physics, chemistry, and beyond, Rev. Mod. Phys. 89, 015006 (2017).

[20] M. Haelterman, S. Trillo, and S. Wabnitz, Dissipative modulation instability in a nonlinear dispersive ring cavity, Opt. Commun. 91, 401 (1992)

[21] A. S. Manka, H. M. Doss, L. M. Narducci, P. Ru, and G.-L. Oppo, Spontaneous emission and absorption properties of a driven three-level system. II. The $\Lambda$ and cascade models, Phys. Rev. A 43, 3748 (1991).

[22] See Supplemental Material at http://link.aps.org/supplemental/ 10.1103/PhysRevA.105.L011501 for the derivation of Eq. (2).

[23] L. A. Lugiato and R. Lefever, Spatial Dissipative Structures in Passive Optical Systems, Phys. Rev. Lett. 58, 2209 (1987).

[24] N. N. Rosanov, Spatial Hysteresis and Optical Patterns (Springer, Berlin, 2002).

[25] P. Coullet, J. Lega, B. Houchmanzadeh, and J. Lajzerowicz, Breaking Chirality in Nonequilibrium Systems, Phys. Rev. Lett. 65, 1352 (1990).

[26] J. Sampaio, J. Grollier, and P. J. Metaxas, in Magnetism of Surfaces, Interfaces, and Nanoscale Materials, edited by R. E.
Camley, Z. Celinski, and R. L. Stamps (Elsevier, Amsterdam, 2015), Chap. 8, pp. 335-370.

[27] B. Deng, S. Yu, A. E. Forte, V. Tournat, and K. Bertoldi, Characterization, stability, and application of domain walls in flexible mechanical metamaterials, Proc. Natl. Acad. Sci. USA 117, 31002 (2020).

[28] F. Sagués and M. San Miguel, Transient patterns in nematic liquid crystals: Domain-wall dynamics, Phys. Rev. A 39, 6567 (1989).

[29] S. Trillo, M. Haelterman, and A. Sheppard, Stable topological spatial solitons in optical parametric oscillators, Opt. Lett. 22, 970 (1997).

[30] G.-L. Oppo, A. J. Scroggie, and W. J. Firth, From domain walls to localized structures in degenerate optical parametric oscillators, J. Opt. B 1, 133 (1999).

[31] G.-L. Oppo, A. J. Scroggie, and W. J. Firth, Characterization, dynamics and stabilization of diffractive domain walls and dark ring cavity solitons in parametric oscillators, Phys. Rev. E 63, 066209 (2001).

[32] M. Gilles, P.-Y. Bony, J. Garnier, A. Picozzi, M. Guasoni, and J. Fatome, Polarization domain walls in optical fibres as topological bits for data transmission, Nat. Photon. 11, 102 (2017)

[33] B. Garbin, J. Fatome, G.-L. Oppo, M. Erkintalo, S. G. Murdoch, and S. Coen, Dissipative Polarization Domain Walls in a Passive Coherently Driven Kerr Resonator, Phys. Rev. Lett. 126, 023904 (2021).

[34] X. Xue, Y. Xuan, Y. Liu, P. H. Wang, S. Chen, J. Wang, D. E. Leaird, M. Qi, and A. M. Weiner, Mode-locked dark pulse Kerr combs in normal-dispersion microresonators, Nat. Photon. 9, 594 (2015).

[35] P. Parra-Rivas, D. Gomila, E. Knobloch, S. Coen, and L. Gelens, Origin and stability of dark pulse Kerr combs in normal dispersion resonators, Opt. Lett. 41, 2402 (2016).

[36] P. Parra-Rivas, E. Knobloch, D. Gomila, and L. Gelens, Dark solitons in the Lugiato-Lefever equation with normal dispersion, Phys. Rev. A 93, 063839 (2016).

[37] B. Garbin, Y. Wang, S. G. Murdoch, G.-L. Oppo, S. Coen, and M. Erkintalo, Experimental and numerical investigations of switching wave dynamics in a normally dispersive fibre ring resonator, Eur. Phys. J. D 71, 240 (2017).

[38] M. Yu, Y. Okawachi, A. G. Griffith, M. Lipson, and A, L. Gaeta, Mode-locked mid-infrared frequency combs in a silicon microresonator, Optica 3, 854 (2016).

[39] K. Bergmann et al., Roadmap on STIRAP applications, J. Phys. B 52, 202001 (2019). 